\title{
Biological transitions
}

Water freezes and metals phase separate into distinct alloys. Crystals under pressure abruptly alter their molecular structures. Such phase transitions reflect spontaneous transformations of collective organization and are among the most surprising physical phenomena. Phase transitions always reflect some kind of microscale battle between competing kinds of order, or between order and disorder. They define natural fault lines along which natural organization can exist, or change from one form to another.

For equilibrium systems, we have a deep theory of how phase transitions take place. The beautiful theory of critical phenomena reveals that many phase transitions fall into distinct classes of behaviour, and explain why, near transition points, key physical properties are often independent of the system's microscopic details. For systems out of equilibrium, our understanding is more piecemeal, which is too bad, given that most of nature is out of equilibrium.

It would hardly be surprising, for example, if biology made extensive use of phase transitions in ensuring the proper workings of living organisms. Biologists have wondered as much, and identified possible examples, running from the basic structure of the cytoplasm in the interior of a cell to the collective migration of cell tissues during organismal development and wound healing. In a recent review, bioengineers Chiu Fan Lee and Jean David Wurtz of Imperial College London explore a few examples in some detail (preprint at https://arxiv.org/abs/1809.11117; 2018), showing how recent theoretical extensions are helping to demonstrate the broad relevance of phase transitions in fundamental biological functions.

Perhaps nothing is more crucial for living organisms than to maintain precise spatial control over their biochemical processes. The cytoplasmic interior of any cell is hugely complex, and myriad organelles - such as the cell nucleus, the lysosome and the mitochondria - serve as organizing centres, hosting key chemical events. In many of these organelles, a physical membrane enforces separation between the interior and exterior. But this isn't true for all organelles, some of which turn out to be 'membraneless'. There's no evident barrier between inside and outside, yet the separation persists all the same.

One interesting case occurs generally in many sexually reproducing organisms. In such organisms, the cells in a developing embryo differentiate into two types, the somatic cells - those that will later develop into tissues, specialized cells of the immune system and the like - and germ cells, which later develop into sperm and egg cells available for reproduction. Germ cell formation requires a stable physical localization of many key molecules, and in most animals this occurs in organelles called 'germ granules', a somewhat unfortunate name as they are more like soft squishy blobs. In the nematode worm, Caenorhabditis elegans, the germ granules are known (for technical reasons) as $\mathrm{P}$ granules, and they contain a complex mixture of RNAs and proteins. Germ cells first emerge when RNA and these $P$ granules localize to the posterior of the one-cell embryo.

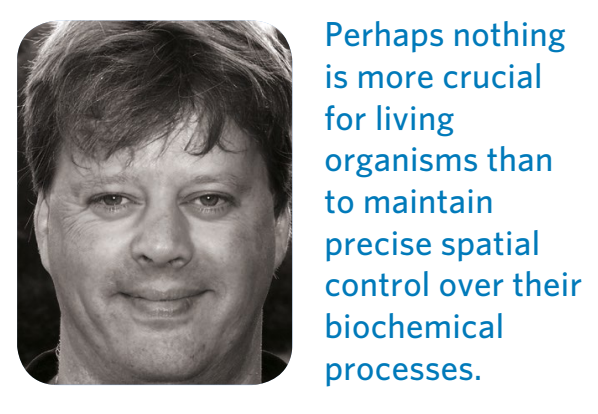

What drives the constituent molecules into formation of a P granule, and what maintains this localization in the absence of a membrane? As Lee and Wurtz point out, experiments suggest that these organelles assemble through a phase transition specifically, a liquid-liquid phase separation, much like the process responsible for oil drop formation in water. Indeed, these organelles even resemble liquid drops in that they are generally spherical and fuse together on contact. The qualitative similarity is striking. But is this really a phase transition?

Phase separation as it occurs in equilibrium isn't quite rich enough to account for what happens, especially the persistence of multiple droplets or independent $\mathrm{P}$ granules. Such an outcome is unstable in equilibrium, as the phenomenon of Ostwald ripening should cause large drops to grow and small drops to evaporate, while any two granules making contact should fuse. Eventually, there should be only one droplet, or the system should have separated completely into two distinct regions, very unlike what happens in reality. However, as Lee and Wurtz demonstrate, simple models of phase separation under non-equilibrium conditions yield very different results. Under normal conditions, the cellular interior is driven away from equilibrium as a result of energy-consuming processes such as ATP-driven protein phosphorylation.

Lee and Wurtz consider a simplified model for a liquid containing three distinct chemical species. To generalize over the equilibrium situation, they suppose that two of the species, A and B, can be transformed by a energy-consuming chemical process. As a result, an A molecule will turn into a $B$ molecule, or vice versa. If driven out of equilibrium, simulations of this model show that phase separation spontaneously occurs, as A-rich drops first appear and grow. Later, Ostwald ripening triggers a gradual growth in the average drop radius until a steady state is reached, determined by the energetic flow into the system. The resulting multidrop system remains stable, in close correspondence with what is seen for $\mathrm{P}$ granule droplets in real cells.

As Lee and Wurtz note, similar phase transitions appear also to lie behind the spontaneous formation of so-called stress granules, other dense liquid agglomerations of proteins that emerge within cells of all types during episodes of stress. Like germ granules, stress granules bring together into one small region many distinct molecules necessary to achieve key tasks for the stressed organism, and do so without any container acting to hold the contents. Phase separation as an alternative to a physical boundary seems to be a trick of quite general use in biology.

Of course, we well know that evolution will make use of any organizing mechanism it discovers. And the authors go on to consider some other biological phase transitions, especially in the behaviour of so-called active matter, such as bacterial swarming over a hard surface. Although these results seem more difficult to support with actual experimental evidence, it appears that researchers have barely begun identifying the many ways biology puts phase transitions to good use.

\section{Mark Buchanan}

Published online: 2 January 2019

https://doi.org/10.1038/s41567-018-0393-4 